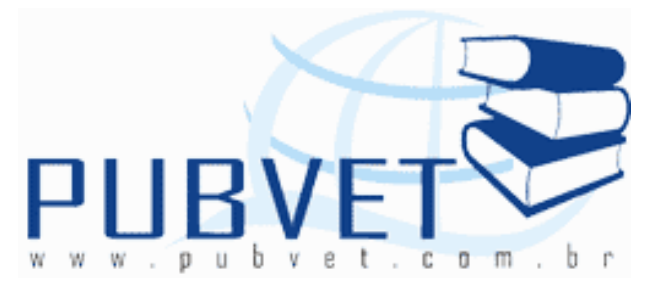

PUBVET, Publicações em Medicina Veterinária e Zootecnia.

\title{
Fratura mandibular em cão atendido no Hospital Veterinário de Uberlândia - Relato de caso
}

\author{
Flávia Resende Martins da Costa ${ }^{1}$, Ananda da Costa Viana ${ }^{2}$, Driene Bastos \\ Soares², Renata Dias Rodrigues², Juliana Capello Toledo², Rafael Rocha de
} Souza ${ }^{3}$

${ }^{1}$ Médica Veterinária. Professora da Faculdade de Medicina Veterinária Universidade Federal de Uberlândia - MG.

${ }^{2}$ Graduandos em Medicina Veterinária. Universidade Federal de Uberlândia MG.

${ }^{3}$ Residente de Patologia Clínica Veterinária. Universidade Federal de Uberlândia - MG.

\section{Resumo}

Fraturas de mandíbula e maxila são comuns em cães e gatos, representando $3 \%$ de todas as fraturas em cães e $15 \%$ das que ocorrem em gatos, a principal etiologia dos traumatismos geralmente são os atropelamentos, quedas e brigas. Muitos tratamentos são propostos para a correção desse defeito, como o uso de pino intramedular, a fixação esquelética externa, a cerclagem e o uso de acrílicos e placas ósseas. O presente estudo relata o caso de um cão da raça Poodle, adulto, apresentando fraturas na mandíbula, com perda óssea, além de lesão ocular, causados por mordeduras de outro animal. A técnica utilizada neste foi a transfixação com pino intramedular liso de Kirschner para a região 
rostral, associada à placa óssea para a área do corpo mandibular. O tratamento prescrito no período pós-operatório foi a base de antibiótico, antiinflamatório e analgésico e dieta pastosa. A recuperação total do animal se deu após 30 dias de procedimento cirúrgico.

Palavras-chave: cirurgia, cavidade oral, traumatismos.

\title{
Mandibular fractures in dog taken care in the Uberlândia- Veterinarian Hospital: case report
}

\begin{abstract}
Fractures of the mandible and maxilla are common in dogs and cats, representing $3 \%$ of all fractures in dogs and $15 \%$ of which occur in cats, the main cause of injuries are usually the motor vehicle collisions, falls and fights. Many treatments are proposed to correct this defect, as the use of intramedullary pin, the external skeletal fixation, cerclage and use of acrylics and bony plates. The present study reports the case of a dog's Poodle Adult, showing fractures in the jaw with bone loss, and ocular injury caused by bites from another animal. The technique used in this was transfixed with Kirschner intramedullary pin flat rostral to the region, coupled with the bone plate to the area of the mandibular body. The treatment prescribed in the postoperative period was the basis of antibiotic, anti-inflammatory and analgesic and past diet. The total recovery of the animal was given 30 days after surgery.
\end{abstract}

Keywords: surgery, oral cavity, injuries.

\section{INTRODUÇÃO}

A face é constituída por um conjunto de ossos que articulam firmemente entre si, apresentando como único osso móvel a mandíbula. A mandíbula participa de funções básicas como mastigação, fonação, deglutição e manutenção da oclusão dentária. Apesar de ser o osso mais pesado e forte da face, a mandíbula está propensa a fraturas, tanto devido a sua anatomia topográfica quanto por ser passível de atrofia com avanço da idade. No que concerne à 
COSTA, F.R.M. et al. Fratura mandibular em cão atendido no Hospital Veterinário de Uberlândia - Relato de caso. PUBVET, Londrina, V. 5, N. 40, Ed. 187, Art. 1262, 2011.

anatomia e topografia, trata-se de um arco aberto projetado no terço inferior da face, freqüentemente atingido por traumas decorrentes de atropelamentos, brigas, quedas, doenças periodontais e neoplasias (UMPLET at al., 1990; PATROCINIO et al., 2005). As fraturas mandibulares são comuns (NUMAMAKER, 1985; TAYLOR, 1990), representando $3 \%$ de todas as fraturas em cães e $15 \%$ das que ocorrem em gatos - PIERMATTEI, 1997). Devido à variedade de inserções musculares presentes na mandíbula, esta oferece aspecto peculiar quanto à possibilidade de fragmentação óssea em alguns tipos e locais de fratura (FURTADO, 1995; TOLEDO et al., 1998; VASCONCELOS et al., 2001). Entre as fraturas de mandíbula, as da região anterior têm grande incidência; o fato de essa região ser anatomicamente projetada para frente das demais estruturas da face faz com que seja extremamente vulnerável a todos os tipos de trauma. Além disso, devem-se considerar outros fatores, tais como a intensidade, a direção do trauma e a presença de grande força de torção encontrada nessa região (NOSÉ \& KURAMOCHI, 2006). Aproximadamente $22 \%$ de todas as fraturas mandibulares são sinfisárias (PETENSON et al., 2000). Segundo (HARVEY \& EMILY, 1993 a maioria das fraturas de mandíbula nos cães acomete a região do corpo mandibular e, nos gatos, a sínfise mentoniana. A maioria das fraturas mandibulares é exposta, podendo ser uni ou bilateral (AMARAL, 2008). Como os traumas mandibulares ocorrem, geralmente, em conseqüência de brigas ou por traumatismos veiculares, na maior parte dos casos as fraturas apresentam-se abertas e contaminadas BOUDRIEAU, 2004; LEGENDRE, 2005; LOPES et al., 2005). Dentre os métodos para fixação de fraturas do corpo mandibular estão o uso de pinos intramedulares, fixadores externos, placas, fios metálicos e incorporação de acrílico

DULISCH, 1985; TAYLOR, 1990; DAVISON, 1993; EGGER, 1993; PIERMATTEI \& FLO, 1997). Os fixadores externos são úteis para estabilizar fraturas não unidas, múltiplas, bilaterais e instáveis, sendo geralmente bem tolerados (PIERMATTEI \& FLO, 1997). Requerem mínima dissecação e há pouca interrupção do fluxo sanguíneo local, proporcionando um ambiente que estimula a cicatrização (EGGER, 1993). As placas são ideais para fraturas 
COSTA, F.R.M. et al. Fratura mandibular em cão atendido no Hospital Veterinário de Uberlândia - Relato de caso. PUBVET, Londrina, V. 5, N. 40, Ed. 187, Art. 1262, 2011.

complexas, proporcionando boa rigidez (EGGER, 1993; PIERMATTEI \& FLO, 1997). Conseguem manter a redução anatômica perfeita e a estabilidade do local de fratura com oclusão normal (NUNAMAKER, 1985). O tratamento cirúrgico de fraturas sinfisárias é facilitado pela ausência de estruturas anatômicas nobres abaixo dos ápices dos dentes inferiores anteriores aos forames mentoais (ELLIS III \& GHALI). A consolidação óssea adequada depende de alguns fatores que são: estabilidade adequada, alinhamento oclusal, preservação de tecidos moles e duros e da dentição que por sua vez vão culminar com o retorno imediato da função. São nesses pontos básicos que a reparação das fraturas dos ossos da maxila e da mandíbula são fundamentados.

\section{RELATO DE CASO}

Foi encaminhado ao Centro Cirúrgico do Hospital Veterinário da Universidade Federal de Uberlândia um cão da raça Poodle, adulto, apresentando fraturas na mandíbula, com perda óssea, além de lesão ocular, causados por mordeduras de outro animal. Como medicação pré-anestésica utilizou-se, por via intramuscular, diazepam como tranqüilizante e cloridrato de xilazina, a fim de promover o miorelaxamento desejado. Foram ainda administrados cloridrato de tramadol $(4 \mathrm{mg} / \mathrm{Kg})$ como analgésico, cetoprofeno $(2 \mathrm{mg} / \mathrm{Kg})$ como antinflamatório e cefazolina $(30 \mathrm{mg} / \mathrm{Kg})$ como antibiótico, todos por via intravenosa. A indução e manutenção anestésica baseou-se em halotano em oxigênio através da via inalatória e a fluidoterapia intravenosa trans-operatória foi feita com solução fisiológica de $\mathrm{NaCl}$ a $0,9 \%$. Para a redução das fraturas utilizou-se técnica de transfixação com pino intramedular liso de Kirschner para a região rostral, associada à placa óssea para a área do corpo mandibular. As extremidades do pino foram dobradas e protegidas com polimetilmetacrilato (Figura 1-6).

Prescreveu-se para o período pós-operatório cefalexina, cloridrato de tramadol e cetoprofeno, bem como dieta pastosa. A recuperação total do animal se deu após 30 dias de pós-operatório. 
COSTA, F.R.M. et al. Fratura mandibular em cão atendido no Hospital Veterinário de Uberlândia - Relato de caso. PUBVET, Londrina, V. 5, N. 40, Ed. 187, Art. 1262, 2011.

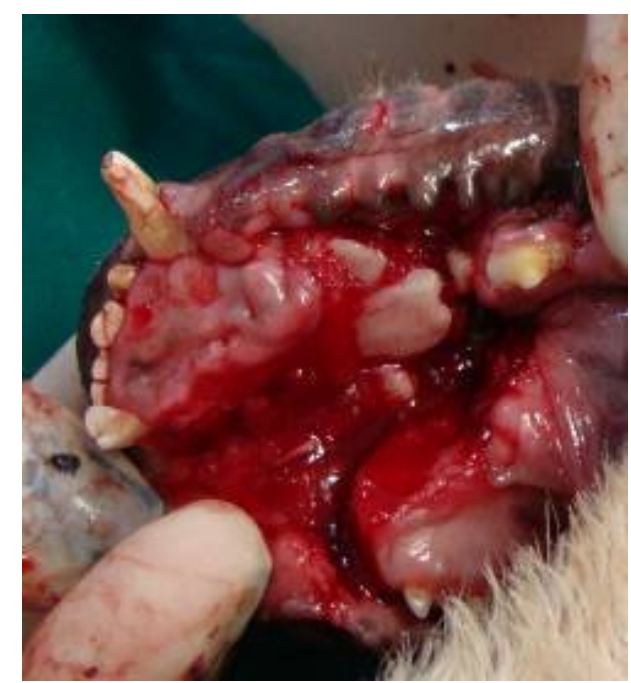

Figura 1: Fraturas na mandíbula. Hospital Veterinário da Universidade Federal de Uberlândia. Arquivo pessoal, 2010.

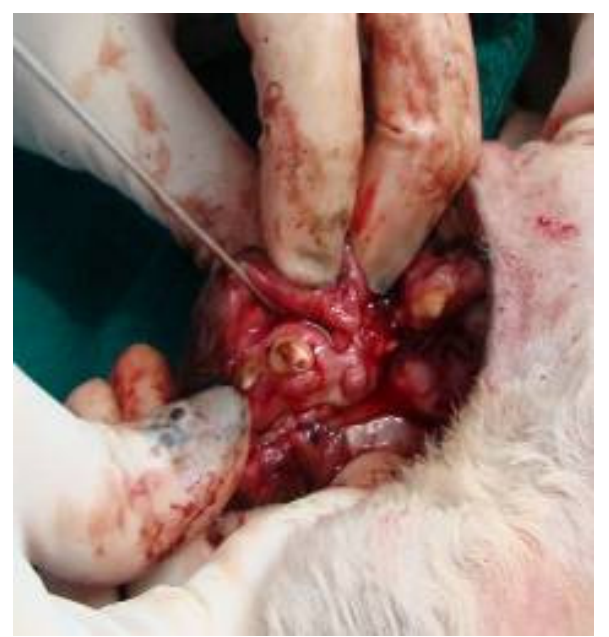

Figura 2: Inicio da redução com pino intramedular liso de Kirschner. Hospital Veterinário da Universidade Federal de Uberlândia. Arquivo pessoal, 2010 
COSTA, F.R.M. et al. Fratura mandibular em cão atendido no Hospital Veterinário de Uberlândia - Relato de caso. PUBVET, Londrina, V. 5, N. 40, Ed. 187, Art. 1262, 2011.

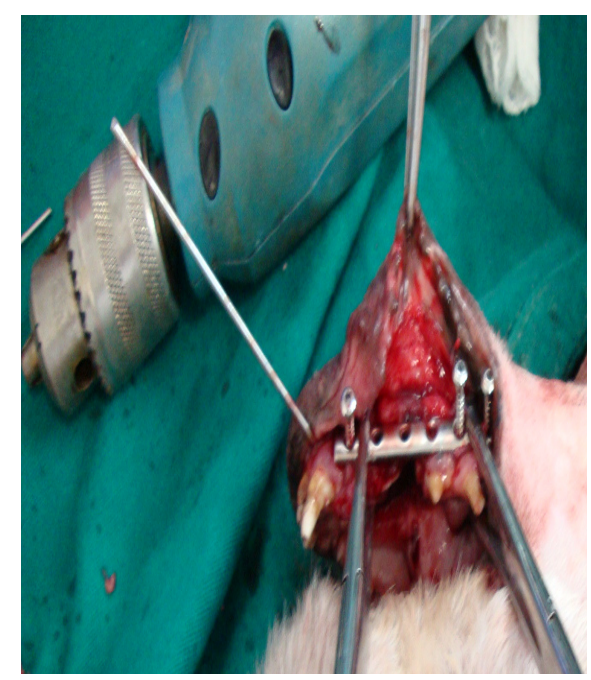

Figura 3: Posicionamento da placa óssea no corpo mandibular. Hospital Veterinário da Universidade Federal de Uberlândia. Arquivo pessoal, 2010.

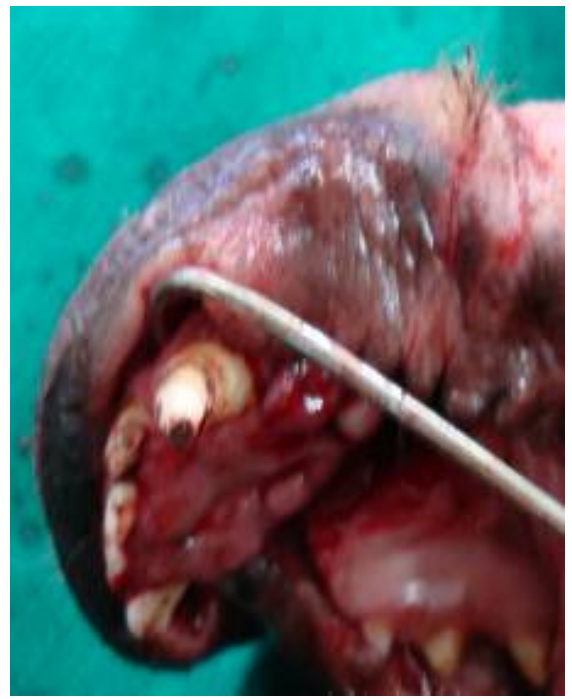

Figura 4: Extremidades dos pinos. Hospital Veterinário da Universidade Federal de Uberlândia. Arquivo pessoal, 2010. 
COSTA, F.R.M. et al. Fratura mandibular em cão atendido no Hospital Veterinário de Uberlândia - Relato de caso. PUBVET, Londrina, V. 5, N. 40, Ed. 187, Art. 1262, 2011.

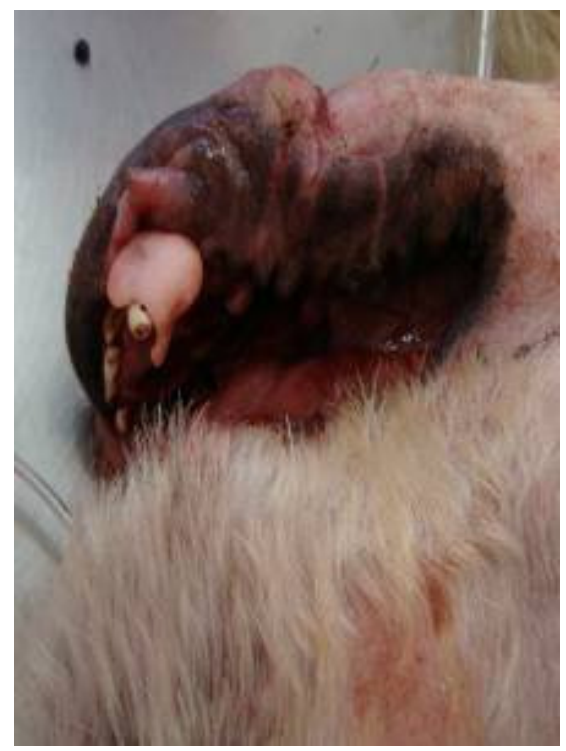

Figura 5: Proteção dos pinos com polimetilmetacrilato. Hospital Veterinário da Universidade Federal de Uberlândia. Arquivo pessoal, 2010.

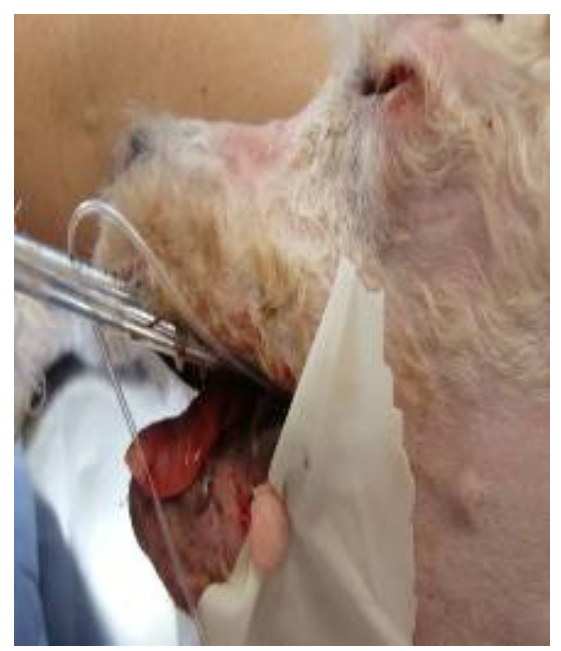

Figura 6: Aspecto final da cirurgia. Hospital Veterinário da Universidade Federal de Uberlândia. Arquivo pessoal, 2010. 
COSTA, F.R.M. et al. Fratura mandibular em cão atendido no Hospital Veterinário de Uberlândia - Relato de caso. PUBVET, Londrina, V. 5, N. 40, Ed. 187, Art. 1262, 2011.

\section{DISCUSSÃO}

Os casos de fraturas de mandíbula são comumente vistos na clinica de pequenos, o procedimento cirúrgico em cães e gatos devem obedecer quatro princípios basicos: oclusão adequada, consolidação óssea perfeita, alinhamento e funcionalidade. Preconiza-se que para fratura de mandíbula sem presença de deslocamento ósseo o tratamento não cirúrgico, sendo aplicado meio conservador obedecendo os princípios de imobilização por meio de fitas e comumente usado em separação de sínfise ou fratura unilateral de mandíbula onde temos boa estabilidade (FURTADO, 1995; HULSE \& JOHNSON, 1997; EGGER, 1998). Porém quando temos fraturas sem alinhamento com oclusão comprometida e presença de fragmentos ósseo, os meios invasivos fazem-se necessários. A placa óssea e parafuso são indicadas para redução de fraturas complexas e bilaterais, proporciona excelente estabilização da fratura permitindo uso das arcadas imediatamente após a cirurgia (HULSE \& JOHNSON, 1997; PIERMATTEI \& FLO, 1997; EGGER, 1998). O aparelho de Kirschner Ehmer é o mais utilizado, contudo, sua aplicação é limitada devido ao custo. 0 uso de acrílico é uma forma de substituir as braçadeiras convencionais é de fácil colocação, conferindo boa estabilidade, conforto ao animal e diminuir o custo do material O principio da colocação segue os utilizado em qualquer tipo de fixador externo (DAVIDSON \& BAUER, 1992; GUEIROS \& BORGES, 1999).

\section{CONCLUSÃO}

A escolha de um método de estabilização nas fraturas mandibulares depende da particularidade do caso e da apresentação da lesão, considerando-se a familiaridade do cirurgião com a peculiaridade e complexidade da técnica. A associação da técnica de transfixação com pino intramedular liso de Kirschner em conjunto com à placa óssea mostraram-se eficientes para a manutenção da estabilidade das fraturas, permitindo a consolidação óssea, bem como a 
COSTA, F.R.M. et al. Fratura mandibular em cão atendido no Hospital Veterinário de Uberlândia - Relato de caso. PUBVET, Londrina, V. 5, N. 40, Ed. 187, Art. 1262, 2011.

alimentação voluntária dos pacientes, sem perda de peso e com o mínimo de complicações.

\section{REFERÊNCIAS}

AMARAL, V. C. do. Fraturas de mandíbula - relato de caso de disjunção de sínfise mandibular em felino. 74f. Monografia (Especialização em Odontologia Veterinária) - Associação Nacional de Clínicos Veterinários de Pequenos Animais de São Paulo e Universidade Anhembi-Morumbi, São Paulo, SP, 2008.

BOUDRIEAU, R.J. Miniplate reconstruction of severely comminuted maxillary fractures in two dogs. Vet. Sur. v.33, p.154-163, 2004.

BRINKER, PIERMATTEI, D. L.; FLO, R. L. Ortop e tratamento das frat peq anim. São Paulo: Manole, 1999.

DAVIDSON, J. R.; BAUER, M. S. Fractures of the Mandible an Maxilla. Vet Clin North Amer: Small Anim Prac, v. 22, n. 1, p. 109-119, 1992.

DAVISON, J.R. tratamiento de las fracturas mandibulares y maxilares em el perro y el gato.

Waltham Inter Focus, v. 3, p. 9-16, 1993.

DULISCH, M. L. Skull and mandibular fractures. In: SLATTER, D. Textbook of Small Anim Surg. Philadelphia: Saunders, v. 2, p. 2286-2295. 1985.

EGGER, E. L. Fraturas de Crânio e Mandíbula. In: SLATTER, D. Manual de Cirurgia de Pequenos Animais. 2. ed. São Paulo: Manole, p. 2253-2265, 1998.

EGGER, E.L.Skull and mandibular fractures. In: SLATTER, D. Textbook of Small Anim Surg. 2.ed. Philadelphia: Saunders, v.2, p.1910-1920, 1993.

ELLIS III, E.; GHALI, G. E. Lag screw fixation of anterior mandibular fractures. J Oral Maxillofacial Surg, v. 49, p. 13-21, 1991.

FURTADO, J. H. C. Frat Bucomaxilofaciais. São Paulo: Pancast, 1995, 123p.

GUEIROS, V. A.; BORGES, A. P. B. Fixação esquelética externa e sua aplicação em pequenos animais. Rev Clín Vet, ano IV, n. 22, p. 31-36, 1999.

HARVEY, C.E., EMILY, P.P. Small anim dent. St. Louis: Mosby, 413 p. 1993.

HULSE, D. A.; JOHNSON, A. L. Tratamento de Fraturas Específicas. In: FOSSUM, T. W. Cir Peq Anim. 2 ed. São Paulo: Roca, cap. 29, p. 854-866, 1997.

LEGENDRE, L. Maxillofacial Fracture Repairs. Veterinary Clinics of North America: Small Anim Pract. v. 35, p. 985-1008, 2005.

LOPES, F.et al. Oral fractures in dogs of Brazil - a retrospective study. J Vet Dent. v. 22, p. 86-90, 2005.

NOSÉ, F. R.; KURAMOCHI, M. M. Técnica Lag screw (LS) para tratamento das fraturas da região anterior da mandíbula. Com Sientia e Saúde, São Paulo, v. 5, p. 51-57, 2006. 
NUNAMAKER, D. M. Fractures and dislocations of the mandible. Im: NEWTON, C. D., NUNAMAKER, D. M. Textbook os small animal orthopaedics. Philadelphia: Lippincott, cap. 18, p. 297-305, 1985.

PATROCÍNIO, L. G. et al. Fratura de mandíbula: análise de 293 pacientes tratados no Hospital de Clínicas da Universidade Federal de Uberlândia. Rio de Janeiro: Rev Bras Otorrinolaringol.v.71, p. 560-565, 2005.

PETENSON, L. J. et al. Cirurgia oral e maxilofacial contemporânea. 3. ed. Rio de Janeiro: Guanabara Koogan; 2000.

PIERMATTEI, D. L., FLO, G. L. Brinker, Piermattei and Flo's Handbookof Small Anim Orthop and Fract Rep. 3. ed. Philadelphia: Saunders, cap. 20: Fractures and luxations of the mandible and maxilla: p. 659-675, 1997.

RAIMUNDO, R.C. Fraturas de mandíbula: análise retrospectiva de 27 casos. Rev Cir e Traumat Buco-Maxilo-Fac, v.8, n.1, p.57-62, 2008. Disponível em: http://www.revistacirurgiabmf.com/2008/v8n1/8.pdf>. Acesso em: 29 jul. 2010.

TAYLOR, R. A. Mandibular fractures. In: BOJRAB, M. J., BIRCHARD, S.J., TOMLINSON, J.L. Cur tec in small anim surg. Philadelphia: Lea \& Febiger, 1990, p. 890-894.

TOLEDO, F. et al. Utilizaçäo de miniplacas no tratamento de fraturas da mandíbula. Rev. Assoc. Paul. Cir. Dent, v.52, p.55-62, 1998.

UMPLET, R.C.; JOHNSON, A.L. Mandibular fractures in the dog. A retrospective study of 157 cases. Vet Surg v.19, p. 272-275, 1990.

VASCONCELLOS, R.J.H. et al. Métodos de tratamento das fraturas mandibulares, Rev.

Cir.Traumat. Buco-maxilo-facial, v.1, p.21-27, 2001. 